Groupement Hospitalier Pitié-Salpêtrière, Département d'Immunologie, Paris, France

Background: The understanding of Erdheim-Chester Disease (ECD) pathogenesis has been greatly improved these past few years with the discovery of activating MAPK pathway mutations in most of ECD patients. However, the inflammatory phenotype of ECD remains widely unknown.

Objectives: We aimed to explore the inflammatory phenotype of Erdheim-Chester disease (ECD) using mass cytometry.

Methods: We analyzed peripheral blood mononuclear cells from 13 ECD patients and 11 healthy donors (HD) using mass cytometry with 29 metal-conjugated antibodies.

Results: Compared to HD, untreated ECD patients had increased proportion of classical monocytes (90.8 [87.1-96.5] vs 81.6 [76.2-87.5] \%, $p=0.02$ ) and decreased proportion of non-classical monocytes (4.7 [3.4-9.7] vs 11.8 [6.617.2] $\%, p=0.047)$. Untreated ECD patients had more circulating Th17 cells compared to HD (3.3 [3-5.3] vs 1.3 [0.4-2.3] \%, $p=0.015)$ and ECD patients treated with BRAF or MEK inhibitors (3.3 (3-5.3] vs 1.9 [0.6-2.4] \%, $\mathrm{p}=0.005)$. Moreover Treg cells were lower in ECD patients than HD, with an increased Th17/Treg ratio (1.37 [0.74-1.9] vs 0.34 [0.19-0.43], $\mathrm{p}=0.0004)$. There was no difference regarding Th1 cells, Th2 cells, B cells, NK cells and circulating dendritic cells.

Conclusion: ECD monocyte profile seems similar to what have been described in CMML. Inflammation observed in ECD may be driven through Th17 cells, and might be targeted with specific treatment.

Disclosure of Interests: None declared

DOI: 10.1136/annrheumdis-2020-eular.3705

\section{AB0039 ROLE OF MESENCHYMAL STEM CELLS ISOLATED FROM DENTAL PULP (DPSCS) IN IMMUNOREGULATION PROCESSES MEDIATED BY PROGRAMMED DEATH-LIGAND 1 (PD-L1)}

E. Pignatti ${ }^{1}$, A. Pisciotta ${ }^{1}$, G. Bertani ${ }^{1}$, R. Di Tinco ${ }^{1}$, L. Bertoni ${ }^{1}$, S. Croci ${ }^{2}$, M. Bonacini ${ }^{2}$, P. Azzoni ${ }^{1}$, A. De Pol ${ }^{1}$, C. Salvarani ${ }^{1,3}$, G. Carnevale ${ }^{1}$. ${ }^{1}$ University of Modena and Reggio Emilia, Department of Surgery, Medicine Dentistry and Morphological Sciences with Interest in Transplant, Modena, Italy; ${ }^{2}$ Azienda Unità Sanitaria Locale di Reggio Emilia-IRCCS, Clinical Immunology, Allergy and Advanced Biotechnology Unit, Reggio Emilia, Italy; ${ }^{3}$ Azienda OspedalieroUniversitaria Policlinico di Modena, Rheumatology Unit, Modena, Italy

Background: Stem cells isolated from dental pulp (DPSCs) are characterized by a high rate of proliferation, low immunogenicity and a high ability to differentiate in different lineages (i.e. osteogenic, chondrogenic, adipogenic, myogenic and neural commitment). Their multipotency can be attributed to the peculiar embryological origin from the neural crest. DPSCs represent a promising stem cell resource since they hold a low ethical impact and can be easily isolated through routine dental procedures. These cells own immuno-modulatory properties, exerted through the activation of different mechanisms, including the Fas / FasL pathway, as well as through the release of soluble factors. Currently, other molecular mechanisms are under consideration such as PD-1 / PD-L1 (Programmed Death 1 and its Ligand) which are supposed to be involved in the induction and / or maintenance of immune tolerance.

Objectives: The aim of this research was to investigate whether the stimulation of PD-L1 in DPSCs can affect the immunomodulatory effects of these stem cells on peripheral blood mononuclear cells (PBMCs). Furthermore, the expression of PD-L1 was also assayed after the induction of osteogenic differentiation of DPSCs in order to evaluate a possible application of DPSCs in autoimmune inflammatory osteo-erosive diseases.

Methods: Immuno-selection was performed on DPSCs, isolated from waste material, against the stemness markers c-Kit and STRO-1, to obtain a pure stem cell population. Then, STRO-1+/c-Kit+ DPSCs, were co-cultured either directly and indirectly with peripheral blood mononuclear cells (PBMCs) from healthy adult donors, previously activated by anti-CD3 and anti-CD28 antibodies. Cocultures of PBMCs with amniotic fluid stem cells (AFSCs) and bone marrow mesenchymal stem cells (BM-MSCs) were also set up. The expression of PD-1 in PBMCs as well as of PD-L1 in DPSCs, AFSCs, BM-MSCs and PBMCs, was evaluated by Western Blot (WB) and immunofluorescence (IF) analyses, before and after osteogenic differentiation. Osteogenic differentiation of DPSCs, after 30 days of induction, was verified by IF and WB, of osteopontin, osteocalcin and RUNX2 markers. Interleukin-2 (IL-2) expression levels in PBMCs were analyzed by Real-Time PCR analysis.

Results: Our data highlight that, after direct and indirect co-culture with activated PBMCs, PD-L1 expression was up-regulated not only in DPSCs, but also in BM-MSCs and AFSCs (Figure 1), thus suggesting that 1) this is a common ability of mesenchymal stem cells and 2) this event can be also mediated by soluble factors release. Moreover, when evaluating the effects of DPSCs co-culture on PBMCs an increased expression of cleaved caspase 3 was observed, together with a decreased expression of IL-2 - a growth factor essential for the proliferation and survival of $T$ cells (Figure 2). These findings showed how DPSCs can modulate the immune system by PD-L1 up-regulation. On the other hand, it is noteworthy that, after reaching osteogenic commitment, DPSCs down-regulated the expression of PD-L1, allowing to hypothesize that PD-L1 expression is strictly related to the maintenance of stemness.
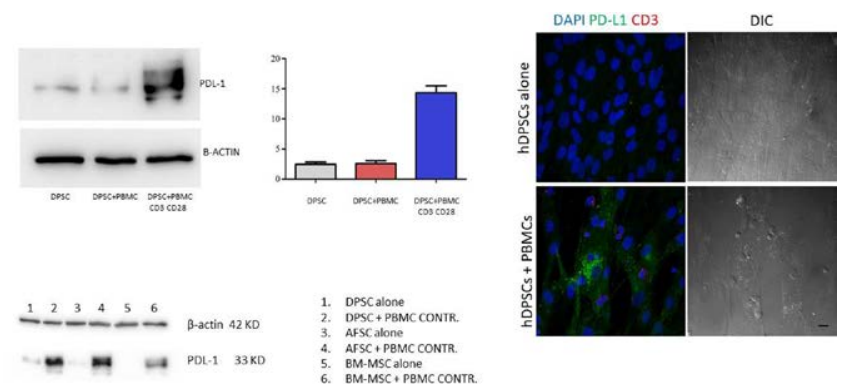

Figure 1.
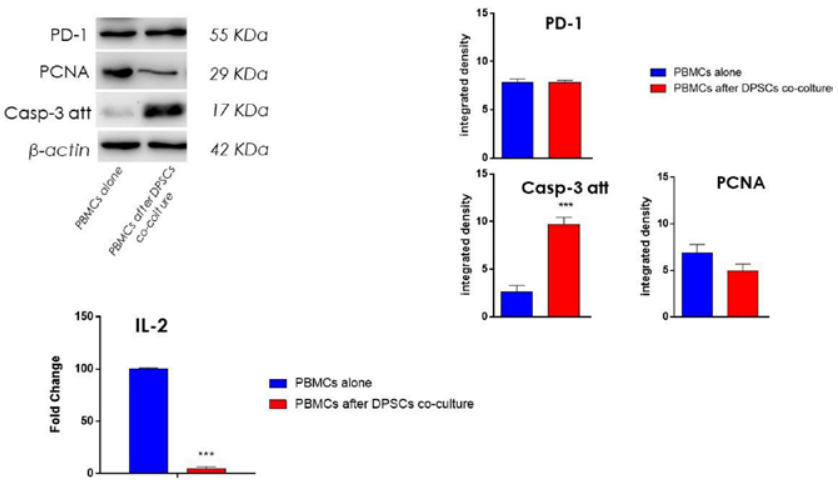

Figure 2.

Conclusion: Taken together, our findings suggest that the expression of PD-L1 in DPSCs is involved in the modulation of immune response and pave the way for further investigations on the role of PD-1/PD-L1 pathway in controlling inflammation and immune response when applied to the treatment of autoimmune inflammatory diseases.

\section{References:}

[1] Keir ME, et al. PD-1 and its ligands in tolerance and immunity. Annu Rev Immunol. 2008

Disclosure of Interests: None declared

DOI: 10.1136/annrheumdis-2020-eular.3696

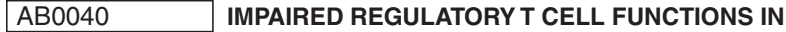 PATIENTS WITH PSORIASIS ARTHRITIS ELIGIBLE TO SWITCH TO ANTI-IL-17 TREATMENT}

T. Christoforou ${ }^{1}$, G. Almanzar ${ }^{1}$, F. Brauneiser ${ }^{1}$, N. Buschmann ${ }^{1}$,

M. Feuchtenberger ${ }^{2}$, M. Schmalzing ${ }^{3}$, H. P. Tony ${ }^{3}$, M. Goebeler ${ }^{4}$, M. Prelog ${ }^{1}$.

${ }^{1}$ University of Wuerzburg, University Children's Hospital, Pediatric

Rheumatology/Special Immunology, Wuerzburg, Germany; ${ }^{2}$ Department of

Medicine II, Division of Rheumatology and Clinical Immunology, Altoetting-

Burghausen, Germany; ${ }^{3}$ University Hospital Wuerzburg, Department of

Medicine II, Rheumatology and Clinical Immunology, Wuerzburg, Germany;

${ }^{4}$ University Hospital Wuerzburg, Department of Dermatology, Wuerzburg,

Germany

Background: A dysbalance between Th17 and regulatory T cells (Treg) has been suggested for several T cell-mediated autoimmune disorders. Inhibitors of $\mathrm{IL}-17$ are successfully used for treatment of psoriasis arthritis (PsA). However, so far reconstitution of Treg functions has not been studied in detail in PsA eligible for switching to anti-IL-17 treatment.

Objectives: The project aims to analyze the reconstitution and maintenance of regulatory $T$ cell (Treg) function after inhibition of inflammatory Th17-inducing pathways mediated by IL-1, IL-6, IL-17 and TNFalpha in a longitudinal manner.

Methods: Therefore, Treg derived from 12 PsA patients switching to Th17 inhibition and healthy controls were phenotypically characterized by flow cytometry. 
Function was investigated by analysis of suppressive activity of Treg on proliferation of autologous effector T cells in vitro utilizing suppression assays.

Results: First results at the time-point of switching to anti-IL-17 treatment demonstrated PsA to be an IL-17-driven T cell-mediated autoimmune disorder, as proportions of T cells with Th17 phenotype were increased in PsA compared to controls (CCR6+lL-17+4.9\% vs. $0.8 \%$ of $\mathrm{CD} 4+$ ) and FoxP3+ Treg cells (CD25brightFoxP $3+0.2 \%$ vs. $0.4 \%$ of CD4+) were decreased. Higher proportions of FoxP3+ T cells expressing the Th17-characteristic chemokine receptor CCR6 were found in PsA (4.8\% vs. $2.7 \%$ of CD4+), as well as higher proportions of pro-apoptotic CD95-expressing FoxP3+T cells ( $9.8 \%$ vs. $2.8 \%$ of CD4+). Less suppression of autologous effector $\mathrm{T}$ cells co-cultured with CD25+ Treg cells was found in PsA compared to controls $(22.2 \%$ vs. $28.3 \%$ reduction of proliferative activity), whereas CD25- helper $\mathrm{T}$ cells did not contribute to the suppression of effectors in PsA and only minimally in controls. Intracellular IL-10 production in Tregs, a key cytokine of Treg-associated regulation of inflammation, was similar between PsA and controls, although a trend to lower CTLA-4 expression involved in inhibition of co-stimulation was found in PsA.

Conclusion: The current results indicate a skewed $T$ cell balance towards Th17 cells and Treg cells showing Th17-like features in samples of PsA unsuccessfully pre-treated with different biologics recommending them for a switch to a therapy with selective inhibition of IL-17. Longitudinal results regarding the reconstitution and maintenance of Treg function in those PsA patients have to be awaited.

Disclosure of Interests: Timotheos Christoforou: None declared, Giovanni Almanzar Grant/research support from: Pfizer, Franziska Brauneiser: None declared, Nils Buschmann: None declared, Martin Feuchtenberger Consultant of: Abbvie, BMS, Chugai, Sanofi, Speakers bureau: Abbvie, BMS, Celgene, Chugai, Jansen-Cilag, Lilly, Pfizer, Roche, Sanofi, UCB, Marc Schmalzing Consultant of: Paid consultant for Hexal AG, Hans-Peter Tony Consultant of: AbbVie, Astra-Zeneca, BMS, Chugai, Janssen, Lilly, MSD, Novartis, Pfizer, Roche, Sanofi, Matthias Goebeler: None declared, Martina Prelog Grant/research support from: Chugai, Sobi, Novartis, Pfizer, Baxter, Consultant of: GSK, Pfizer, Novartis, MSD, Baxter, Sobi, Johnson, Speakers bureau: GSK, Pfizer, Novartis, MSD, Baxter, Sobi, Johnson DOI: 10.1136/annrheumdis-2020-eular.6389

\section{AB0041 CD8+ T CELLS HAVE AN ELEVATED PROLIFERATIVE CAPACITY IN GIANT CELL ARTERITIS}

R. Reitsema ${ }^{1}$, R. Hid Cadena ${ }^{2}$, W. Abdulahad ${ }^{1,2}$, A. Boots ${ }^{1}$, P. Heeringa ${ }^{2}$, E. Brouwer ${ }^{1}{ }^{1}$ University of Groningen, University Medical Center Groningen, Rheumatology and Clinical Immunology, Groningen, Netherlands; ${ }^{2}$ University of Groningen, University Medical Center Groningen, Pathology and Medical Biology, Groningen, Netherlands

Background: Giant cell arteritis (GCA) is the most frequent form of systemic vasculitis affecting the large- and medium-sized vessels. The involvement of innate immune cells and CD4+ T cells in the pathogenesis of GCA has been extensively studied. Interestingly, recent findings suggest a role for CD8+ T cells in disease development (1). However, CD8+ subsets and their functional capacities have not yet been studied in detail.

Objectives: This study aims to characterize the phenotype and proliferative capacity of CD8+ T cells in newly diagnosed GCA patients and GCA patients in remission compared to healthy age- and sex- matched controls.

Methods: To determine the phenotype of CD8+ T cells in GCA, newly diagnosed, untreated GCA patients (baseline, $n=14$ ), GCA patients in stable glucocorticoid-free remission (GC-FR, $n=10$ ) and age- and sex-matched healthy controls (HCs, $\mathrm{n}=18$ ) were enrolled. Peripheral blood mononuclear cells (PBMCs) were stained with fluorochrome-conjugated antibodies directed against CD3, CD4, CD8, CCR7, CD45RO, Ki-67, CD69 and CD25 and analyzed by flow cytometry. The following differentiation subsets were defined: CD8+ $T$ naive (CD45RO-CCR7+), central memory ( $\left.T_{C M}, C D 45 R O+C C R 7+\right)$, effector memory ( $\left.T_{E M}, C D 45 R O+C C R 7-\right)$ and effector memory re-expressing CD45RA ( $T_{E M R A}$, CD45RO-CCR7-) cells. Secondly, the proliferative capacity of CD8+ T cells was determined in isolated CD3+ T cells of 10 GCA baseline, 10 GCA GC-FR patients and $19 \mathrm{HCs}$ after 5 days of stimulation with plate-bound anti-CD3 or anti-CD3 plus soluble anti-CD28 using a dye-based proliferation assay.

Results: A reduced frequency of $C D 8+T_{E M}$ cells was found in GCA baseline patients compared to $\mathrm{HCs}(\mathrm{p}=0.025)$. Furthermore, a higher frequency of Ki-67+ cells was detected among CD8 $+\mathrm{T}_{\mathrm{EM}}$ cells in GCA baseline patients than in $\mathrm{HCs}$ $(\mathrm{p}=0.0007)$, suggesting a higher proliferative activity in vivo. In addition, in vitro stimulation with anti-CD3 and anti-CD3+anti-CD28 led to higher percentages of divided CD8+ T cells in GCA baseline and GC-FR patients than in HCs $(p<0.05)$. Moreover, the frequencies of CD8+ $T_{E M R A}$ cells and the percentage of divided CD8+ T cells upon CD3 stimulation strongly correlated in GCA baseline patients
$(R=0.79, p=0.009)$ and GCA GC-FR patients $(R=0.67, p=0.039)$ but not in HCs $(\mathrm{R}=0.31, \mathrm{p}=0.25)$

Conclusion: GCA baseline patients demonstrate a higher frequency of proliferating circulating CD8+ $\mathrm{T}_{\mathrm{EM}}$ cells, defined by $\mathrm{Ki}-67$ expression, than $\mathrm{HCs}$. In addition, functional data on induced proliferative capacity suggest that CD8+ $T$ cells from GCA baseline patients are more rapidly activated by crosslinking CD3 and CD3+CD28, suggesting either reduced regulation in these patients or more intrinsic threshold changes. Furthermore, the induced proliferative capacity is also elevated in patients in stable glucocorticoid-free remission. Whether the increased proliferative capacity of total CD8+ T cells in GCA patients is causally linked to the increased frequencies of $C D 8+T_{E M R A}$ cells in these patients requires further investigation.

\section{References:}

[1] Samson M, Ly KH, Tournier B, Janikashvili N, Trad M, Ciudad M, et al. Involvement and prognosis value of CD8+ T cells in giant cell arteritis. J Autoimmun. 2016;72:73-83.

Disclosure of Interests: Rosanne Reitsema: None declared, Rebeca Hid Cadena: None declared, Wayel Abdulahad: None declared, Annemieke Boots Consultant of: Grünenthal Gmbh until 2017, Peter Heeringa: None declared, Elisabeth Brouwer Consultant of: Roche (consultancy fee 2017 and 2018 paid to the UMCG), Speakers bureau: Roche (2017 and 2018 paid to the UMCG)

DOI: 10.1136/annrheumdis-2020-eular.3145

\begin{tabular}{ll}
\hline AB0042 & CYTOKINE NETWORK ELUCIDATED BY THE \\
QUANTIFICATION OF MULTIPLE CYTOKINES IN \\
THE SERUM SEQUENTIALLY SAMPLED FROM RA \\
PATIENTS WHO WERE TREATED WITH BIOLOGIC \\
DMARDS
\end{tabular}

K. Sato ${ }^{1}$, S. Mamada ${ }^{1}$, C. Hayashi ${ }^{1}$, T. Nagashima ${ }^{1}$, S. Minota ${ }^{1} .{ }^{1}$ Jichi Medica University, Division of Rheumatology and Clinical Immunology, Tochigi, Japan

Background: Biologic disease modifying anti-rheumatic drugs (DMARDs) have demonstrated that proinflammatory cytokines such as interleukin (IL-) 6 and tumor necrosis factor (TNF) play important roles in the pathogenesis of rheumatoid arthritis (RA). Other cytokines, such as type I interferons (IFNs), are also implicated in its pathogenesis (ref 1). However, the complete picture of the cytokine network involved in RA remains to be elucidated.

Objectives: By quantifying sets of cytokines in the serum of RA patients before and after treatment with various biologic DMARDs, we sought to determine the effects of drugs on (A) type I IFNs, (B) soluble IL-6 receptors, and (C) other cytokines.

Methods: 52 patients with RA were treated with various biologic DMARDs (tocilizumab (TOC): 16, abatacept (ABT): 15, and TNF inhibitors (TNFi): 21). Serum samples were obtained (1) before, (2) approximately 4 weeks after (3) and approximately 12 weeks after the initiation of treatment. A suspension bead-array system was used for analysis; Bio-Plex Human Cytokine 17-plex Assay kits and Express Custom Panels (Bio-Rad), including IFN- $\beta$, IFN- $\alpha 2$, soluble IL-6 receptor a (sIL6Ra) and gp130 were used.

Results: (1) As expected, the disease activity score 28-joiny count (DAS28) using the erythrocyte sedimentation rate (ESR) significantly decreased in all three groups (TOC, ABT and TNFi) by 12 weeks.

(2) IFN- $\alpha 2$ was barely detected in the serum samples. IFN- $\beta$ seemed to increase slightly in the ABT group, but the increase was not statistically significant.

(3) The levels of sIL6Ra did not change substantially. Those of gp130 decreased slightly but significantly in the TOC group by 12 weeks.

(4) The levels of IL- 6 decreased significantly in the ABT group by 12 weeks. Those in the TNFi group decreased significantly at 4 weeks but not 12 weeks (Fig. 1A) (5) The levels of IL-7 decreased significantly only in the TOC group (Fig. 1B). Conclusion: (1) The biologic DMARDs tested in this study did not significantly affect the serum levels of type I IFNs in this study.

(2) The decrease in gp130 in the TOC group may imply that gp130 is induced by IL-6, although whether this level of decrease has physiological significance is open to question.

(3) Serum IL-6 was significantly decreased in the TNFi group at 4 weeks but not 12 weeks. TNF has been reported to induce IL-6 (ref 2), but negative feedback loop(s) may be present. Such a feedback system might make the discontinuation of TNFi difficult, even if patients are in remission.

(4) IL-7 may be a target of IL-6. A higher level of IL-7 has been reported to be present in the joints of RA patients compared with osteoarthrosis and it is a cytokine implicated in the differentiation of osteoclasts (ref 3 ). This may partly explain the effect of TOC on preventing bone erosion in RA.

References:

[1] Ann Rheum Dis. 2007; 66: 1008-14

[2] Rheumatology $2007 ; 46: 920-6$

[3] Rheumatology 2008; 47: 753-9 